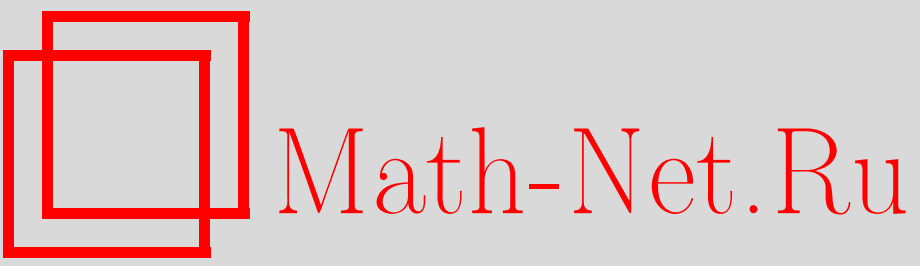

А. Д. Миронов, Матричные модели и матричные интегралы, ТМФ, 2006, том 146, номер 1, 77-89

DOI: https://doi.org/10.4213/tmf2010

Использование Общероссийского математического портала Math-Net.Ru подразумевает, что вы прочитали и согласны с пользовательским соглашением

http://www.mathnet.ru/rus/agreement

Параметры загрузки:

IP : 34.229 .108 .108

26 апреля 2023 г., 13:16:05

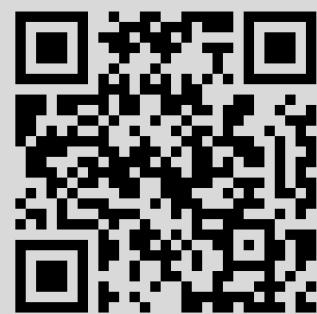


ТЕОРЕТИЧЕСКАЯ

И МАТЕМАТИЧЕСКАЯ

ФИЗИКА

Том 146, № 1

январь, 2006

(C) 2006 г.

А. Д. Миронов*

\title{
МАТРИЧНЫЕ МОДЕЛИ И МАТРИЧНЫЕ ИНТЕГРАЛЫ
}

\begin{abstract}
Обсуждаются взаимосвязи между произвольными решениями петлевых уравнений, описывающих эрмитову одноматричную модель, и частными (многоразрезными) решениями, которые отвечают конкретным матричным интегралам. Последние обладают рядом специальных свойств и, в частности, описываются в терминах теории Зайберга-Виттена-Уизема. В деталях рассмотрен простейший пример обычного интеграла.
\end{abstract}

Ключевые слова: матричные модели, суперсимметричные калибровочные теории.

\section{1. ВВЕДЕНИЕ}

Исследования $\mathcal{N}=1$ суперсимметричных калибровочных теорий в работах Качазо, Интриллигатора и Вафы [1], а также предложение Дийкграафа и Вафы [2] вычислять низкоэнергетические суперпотенциалы, используя статистические суммы, отвечающие многоразрезным решениям, определили недавно возникший интерес к матричным моделям и в особенности к их многоразрезным решениям. Сами эти решения хорошо известны уже давно (см., например, [3]), однако работа Боннэ, Давида и Эйнара [4] вновь привлекла к ним внимание.

Мы рассматриваем эрмитову одноматричную модель, статистическая сумма которой дается интегралом по $N \times N$ эрмитовым матрицам:

$$
Z_{N}(t) \equiv \frac{1}{\operatorname{Vol}_{U(N)}} \int D M \exp \left(\operatorname{tr} \sum_{k} t_{k} M^{k}\right)
$$

Здесь $D M$ - инвариантная мера (Хаара) на эрмитовых матрицах, которая является просто плоской мерой, a $\operatorname{Vol}_{U(N)}$ - объем унитарной группы $U(N)$ [5]. Поскольку подынтегральное выражение в (1) инвариантно по отношению к сопряжению матрицей, можно свести формулу (1) к интегралу по собственным значениям матрицы $M$,

\footnotetext{
* Физический институт им. П. Н. Лебедева РАН, Институт теоретической и экспериментальной физики, Москва, Россия. E-mail: mironov@itep.ru; mironov@lpi.ru
} 
интегрируя угловые переменные. Это можно сделать, использовав формулы из монографии [6]. Результат имеет вид

$$
Z_{N}(T)=\frac{1}{N !} \int \prod_{i} d x_{i} \Delta^{2}(x) \exp \left(\sum_{k, i} t_{k} x_{i}^{k}\right),
$$

где $\Delta(x)$ - определитель Вандермонда, $\Delta(x) \equiv \operatorname{det}_{i, j} x_{i}^{j-1}=\prod_{i>j}\left(x_{j}-x_{i}\right)$.

Тем не менее все еще необходимо определить интегралы (1) и (2). Действительно, нужно фиксировать контуры интегрирования в этих многократных интегралах. Более того, эти контуры интегрирования могут отличаться друг от друга для разных собственных значений. Говоря так, мы, конечно, забываем, что начинали с эрмитовых матриц. Однако это ни в коей мере не существенно для свойств (1) и (2). На самом деле слово "эрмитова" для матричного интеграла не имеет никакого другого смысла, кроме фиксации контура интегрирования. Действительно важно зафиксировать в формуле (1) плоскую меру, и этого достаточно, чтобы получить (2) из (1).

Чтобы определить выражения (1) и (2), можно заменить их квазиклассическим разложением [4], [7]. Иная возможность [8]-[10] - заметить, что они удовлетворяют бесконечному набору петлевых (вирасоровских) уравнений (или уравнений Швингера-Дайсона, или тождеств Уорда) [11], [12]. Действительно, это следствие только плоской меры и инвариантности интеграла по отношению к замене переменных.

Вторая возможность означает, что статистической суммой матричной модели называется любое решение петлевых уравнений. Тогда статистическая сумма - не функция, а формальный $D$-модуль, т.е. полный набор степенных рядов (по переменным $t$ ), удовлетворяющих системе совместных линейных уравнений. Решение уравнений не обязано быть единственным, однако некоторое подходящее аналитическое продолжение по переменным $t$ преобразует одно решение в другое, и на достаточно большом пространстве модулей (констант связи $t$ ) все это вместе может быть представлено, по крайней мере формально, как единый объект. Его мы и называем статистической суммой.

Грубо говоря, различные решения интерпретируются как различные ветви статистической суммы, ассоциированные с различными фазами теории. Далее, решения линейных дифференциальных уравнений могут быть часто представлены интегралами (по спектральным многообразиям), но “контуры" интегрирования остаются нефиксированными: они могут быть общими цепями с комплексными коэффициентами (в случае целых коэффициентов это часто описывается в терминах монодромий, в случае статистической суммы эти коэффициенты не обязаны быть целыми).

Таким образом, в дальнейшем рассмотрении мы различаем матричную модель, которая является набором решений петлевых уравнений, и матричный интеграл, который является именно интегралом с некоторым заданным контуром интегрирования. Заметим, что статистическая сумма матричной модели является произвольной линейной комбинацией базисных матричных интегралов. 
Естественный выбор такого базиса дается многоразрезными решениями (или решениями Дийкграафа-Вафы), упоминавшимися выше. Они выделены специальным свойством изомонодромности, которое позволяет ассоциировать с ними некоторую систему Зайберга-Виттена-Уизема [13], [14], причем соответствующая статистическая сумма имеет представление в виде многоматричного интеграла [4], [7].

\section{2. СЛУЧАЙ МАТРИЦЫ 1 × 1: ПРОСТЕЙШИЙ ПРИМЕР}

Начнем с простейшего примера "матрицы” $1 \times 1$, т.е. матричный интеграл - это простой однократный интеграл. Рассмотрим вначале интеграл с потенциалом самого общего вида и увидим, что можно сделать в этом случае.

2.1. Петлевые уравнения. Итак, начинаем с интеграла

$$
Z\left(\left\{t_{k}\right\}\right)=\int_{C} d x \exp \left(\sum_{k} t_{k} x^{k}\right) .
$$

Вначале мы не фиксируем контур интегрирования $C$, предполагая, однако, что он замкнут или, по меньшей мере, подынтегральное выражение, умноженное на моном $x^{k}$ любой степени, зануляется на концах контура. Тогда можно немедленно получить бесконечную систему уравнений ${ }^{1)}$, которым удовлетворяет наш интеграл. Для этого достаточно рассмотреть интеграл от полной производной

$$
\int_{C} d x \frac{\partial}{\partial x}\left[x^{n+1} \exp \left(\sum_{k} t_{k} x^{k}\right)\right], \quad n \geqslant-1,
$$

который равен нулю. Этот интеграл приводит к набору условий

$$
\sum_{k} k t_{k} \frac{\partial^{k+n} Z}{\partial t_{1}^{k+n}}+(n+1) \frac{\partial Z}{\partial t_{n}}=0, \quad n \geqslant-1,
$$

где $\partial Z / \partial t_{0} \equiv Z$. Заметим, что

$$
\frac{\partial Z}{\partial t_{k}}=\frac{\partial^{k} Z}{\partial t_{1}^{k}}
$$

и, следовательно, первый член суммы в условиях (5) может быть переписан как первые производные по константам связи $t_{k+n}$.

Попробуем найти решение (5) в виде степенного ряда по $t_{k}$ :

$$
Z=c^{(0)}+\sum_{k} c_{k}^{(1)} t_{k}+\sum_{k, l} c_{k l}^{(2)} t_{k} t_{l}+\cdots
$$

Тогда из условия с $n=0$ мы немедленно получаем $c^{(0)}=0$; подобным же образом $c_{k}^{(1)}=0$ и т.д. Это означает, что решение тривиально: $Z=0$. На самом деле это не

\footnotetext{
1) Эти уравнения называются петлевыми уравнениями, условиями Вирасоро, уравнениями Швингера-Дайсона, тождествами Уорда (см. работы [12]).
} 
должно удивлять, так как размерность констант связи $t_{k}$ равна $k$, а размерность $c^{(l)}$ должна быть отрицательной. У нас, однако, не имеется объектов отрицательной размерности.

Для того чтобы получить нетривиальное решение, нам надо разрешить по крайней мере некоторым константам связи $t_{k}$ появляться в знаменателе. Зафиксируем, что несколько первых (скажем, $p$ ) констант не малы, т.е. мы их сдвигаем: $t_{k} \rightarrow T_{k}+t_{k}, \quad k=1, \ldots, p$, и рассматриваем $Z$ как степенной ряд по $t_{k}$, но не по $T_{k}$. Тогда уравнение (5) имеет нетривиальные решения.

Иными словами, мы фиксируем полином $V(x) \equiv \sum_{k}^{p} T_{k} x^{k}$ и рассматриваем интеграл в выражении (3) как пертурбативный по отношению к $t_{k}$ :

$$
Z\left(\left\{t_{k}\right\}\right)=\int_{C} d x \exp \left(V(x)+\sum_{k} t_{k} x^{k}\right),
$$

т.е. вычисляем моменты

$$
\int_{C} d x x^{k} e^{V(x)}
$$

Производящая функция (резольвента) для этих моментов

$$
G(z) \equiv \int_{C} d x \frac{e^{V(x)}}{z-x}=\sum_{k} \frac{1}{z^{k+1}} \int_{C} d x x^{k} e^{V(x)},
$$

где интеграл понимается в смысле главного значения.

2.2. "Матричная" модель. Рассмотрим простой пример кубического потенциала $V(x)$. Сдвигом $x$ можно обратить в нуль квадратичный член в потенциале и, перемасштабируя $x$, сделать $T_{3}$ равным $-1 / 3$. Следовательно, остается единственная существенная переменная, в качестве которой мы выбираем сейчас $T_{1}$. Связи (5) при нулевых временах сводятся тогда к единственному уравнению

$$
\frac{d^{2} Z}{d T_{1}^{2}}+T_{1} Z=0
$$

которое является не чем иным, как уравнением Эйри. Оно имеет два решения вида

$$
Z\left(T_{1}\right)=\int_{C} d x \exp \left(-\frac{x^{3}}{3}-T_{1} x\right)
$$

которые соответствуют двум существенно различным выборам контуров интегрирования $C$ в уравнении (8). Контур должен быть выбран так, чтобы подынтегральное выражение равнялось нулю на его концах, т.е. он должен уходить на бесконечность такую, что $\operatorname{Re} x^{3}>0$. Одна из возможностей - выбрать в качестве $C$ мнимую ось. Это дает стандартную функцию Эйри

$$
\operatorname{Ai}\left(T_{1}\right)=\int_{0}^{\infty} \cos \left(\frac{x^{3}}{3}-T_{1} x\right) d x .
$$


Другое независимое решение уравнения Эйри должно быть ассоциировано с контуром, связывающим другие бесконечности. Скажем, можно выбрать контур, который идет вдоль мнимой оси из $+\infty$ до нуля и затем вдоль положительного луча вещественной оси. Мы называем соответствующую функцию $\mathrm{Ai}_{2}\left(T_{1}\right)^{2)}$. Тогда (общее) решение "матричной" модели в нашем случае есть

$$
Z\left(T_{1}\right)=\xi \operatorname{Ai}\left(T_{1}\right)+\zeta \mathrm{Ai}_{2}\left(T_{1}\right)
$$

где $\xi$ и $\zeta$ - произвольные константы. Теперь можно использовать уравнения (5), чтобы рекуррентно и однозначно итерировать $Z$ как степенной ряд по $t_{k}$.

Таким образом, мы можем определить статистическую сумму “матричной” модели $Z\left(\left\{t_{k}\right\}\right)$ как решение определяющего набора уравнений (5). Тогда полная свобода, которую мы имеем в нашей “матричной” модели, определяется константами $\xi, \zeta$. Выбор этих констант, т.е. выбор формальной суммы различных контуров интегрирования $C$, фиксирует решение "матричной” модели единственным образом.

2.3. "Матричный" интеграл. В противоположность "матричной" модели "матричный" интеграл определен действительно интегралом. Имеющаяся тогда свобода ограничивается выбором контура интегрирования. Следовательно, можно получить базис в пространстве всех решений, выбирая некоторые базисные контуры. Это дает нам базис "матричных" интегралов, снабженных индексом, ассоциированным с набором базисных контуров. Беря затем линейные комбинации этих интегралов, можно получить общее решение “матричной” модели.

Для эффективных вычислений нужно умно выбрать базис контуров интегрирования. Они естественным образом ассоциируются с асимптотическим разложением интегралов. Скажем, имея дело с кубическим потенциалом, лучше выбирать базисные контуры, отвечающие решениям уравнения Эйри, определяемым различными квазиклассическими разложениями. Действительно, построим асимптотическое разложение $(13)$ при больших $T_{1}$. Тогда перевальное уравнение имеет два решения $x= \pm \sqrt{T_{1}}$. В зависимости от выбора контура интегрирования $C$ нужно выбрать одно или другое решение и разложить интеграл вокруг этого решения, чтобы получить асимптотическое разложение.

В общем случае имеется $p-1$ решение перевального уравнения $V^{\prime}(x)=0$ и ровно столько же квазиклассических разложений и базисных контуров.

Теперь для того, чтобы получить $Z$ как функцию всех $t_{k}$, можно использовать две различные стратегии. Прежде всего, можно итерационно решать уравнения (5). Другая возможность - вычислять моменты (9), используя свойства соответствующих "матричных" интегралов, т.е. в кубическом случае функций Эйри.

Существует еще одна возможность, которая никоим образом не проще технически, однако очень пригодится в дальнейшем. Именно, рассмотрим возможность непосредственного вычисления интеграла (8). Этот интеграл не является степенны́м

\footnotetext{
${ }^{2)}$ Разность $\mathrm{Ai}_{2}\left(T_{1}\right)-\mathrm{Ai}\left(T_{1}\right)$ обычно обозначается $\mathrm{Bi}\left(T_{1}\right)$ (см. [15], формула (10.4.33)).
} 
рядом по старшим константам связи. Действительно, так как степень потенциала много выше $p$, имеется гораздо больше возможностей выбора контуров интегрирования (или квазиклассических режимов, или решений перевальных уравнений). Чтобы иметь степенной ряд по старшим константам связи, мы должны ограничить выбор контура интегрирования так, чтобы поведение интеграла при занулении этих старших констант было гладким (степенны́м), в то время как поведение интеграла по отношению к первым $p$ константам связи остается произвольным. Это оставляет нам свободу выбора ровно $p$ различных контуров интегрирования.

Посмотрим, как это работает в кубическом случае. Здесь у нас имеется две возможности выбора контуров интегрирования. Заметим, что можно свести уравнение Эйри (11) к уравнению Бесселя, а значит, его решение выражается через цилиндрические функции. Более конкретно, функция $\sqrt{T_{1}} Z_{1 / 3}\left((2 / 3) T_{1}^{3 / 2}\right)$ решает уравнение Эйри [15], где $Z_{1 / 3}(z)$ - цилиндрическая функция порядка $1 / 3$. Пусть теперь две базисные цилиндрические функции будут функциями Ханкеля первого и второго родов $H^{(1,2)}(z)$ (этот выбор отвечает не $\mathrm{Ai}\left(T_{1}\right)$ или $\mathrm{Ai}_{2}\left(T_{1}\right)$, а их линейным комбинациям). Восстановим также зависимость от всех трех констант связи (и слегка перемасштабируем их для удобства). Тогда

$$
\begin{gathered}
Z\left(T_{1}, T_{2}, T_{3}\right)=\int d x \exp \left(i T_{3} x^{3}-T_{2} x^{2}+i T_{1} x\right)=\sqrt{\eta} Z_{1 / 3}\left(\frac{2}{3} \eta^{3 / 2}\right) \\
\eta \equiv \frac{T_{2}^{2}}{\left(3 T_{3}\right)^{4 / 3}}-\frac{T_{1}}{\left(3 T_{3}\right)^{1 / 3}}
\end{gathered}
$$

и, используя асимптотическое разложение функций Ханкеля, находим, что гладкое поведение при $T_{3} \rightarrow 0$, т.е. $\eta \rightarrow \infty$, отвечает $H^{(2)}(z)$ (см. [16], формула $(7.13 .2)$ ):

$$
\begin{gathered}
H_{1 / 3}^{(2)}(z) \underset{z \rightarrow \infty}{\sim} \sqrt{\frac{2}{\pi z}} \exp \left(-i \frac{4 z-2 \pi \nu-\pi}{4}\right) \sum_{m=0} \frac{(1 / 3, m)}{(2 i z)^{m}} \\
\left(\frac{1}{3}, m\right) \equiv \frac{\Gamma(5 / 6+m)}{m ! \Gamma(5 / 6-m)} .
\end{gathered}
$$

Следовательно, окончательно имеем (здесь мы для удобства полагаем $T_{1}=0$ )

$$
Z\left(T_{1}, T_{2}, T_{3}\right)=\frac{1}{\sqrt{T_{2}}} \sum_{m=0}(-)^{m}\left(\frac{1}{3}, m\right)\left(\frac{27 T_{3}^{2}}{4 T_{2}^{3}}\right)^{m}
$$

т.е. с таким выбором решения $Z$ действительно является степенны́м рядом по $T_{3}$. Можно легко проверить, что коэффициенты степенно́го разложения здесь совпадают с соответствующими моментами интеграла Гаусса. Следовательно, действительно моменты интеграла Гаусса можно вычислять таким способом.

2.4. Резольвента и петлевое уравнение. В завершение обсуждения нашего простейшего примера обсудим свойства резольвенты (10). Начнем с простейшего 
потенциала Гаусса. Тогда резольвента

$$
G(z) \equiv \int_{-\infty}^{\infty} d x \frac{e^{-T_{2} x^{2}}}{z-x}=2 z \int_{-\infty}^{\infty} d x \frac{e^{-T_{2} x^{2}}}{z^{2}-x^{2}}=w\left(\sqrt{T_{2}} z\right), \quad w(z) \equiv \int_{-\infty}^{\infty} d x \frac{e^{-x^{2}}}{z-x}
$$

может быть вычислена двумя разными способами. Прежде всего, можно использовать формулу

$$
\frac{1}{z-x}=\int_{0}^{\infty} d u e^{-u(z-x)}
$$

и, вычисляя далее интеграл Гаусса, выразить резольвенту через интеграл ошибок ${ }^{3)}$ :

$$
\begin{gathered}
w(z)=e^{-z^{2}}\left(-i \pi+2 \sqrt{\pi} \int_{0}^{z} e^{x^{2}} d x\right)=e^{-z^{2}} \operatorname{erfc}(-i z) \\
\operatorname{erfc}(z) \equiv-i 2 \sqrt{\pi} \int_{z}^{\infty} e^{-x^{2}} d x
\end{gathered}
$$

Другая возможность - найти дифференциальное уравнение для $w(z)$. С этой целью снова используем равенство нулю интеграла от полной производной:

$$
0=\int_{-\infty}^{\infty} d x \frac{\partial}{\partial x}\left(\frac{e^{-x^{2}}}{z-x}\right)
$$

Это ведет к уравнению

$$
w^{\prime}(z)=2 \sqrt{\pi}-2 z w(z)
$$

решая которое, мы снова приходим к выражению (20).

В случае более сложных потенциалов $V(x)$ невозможно явно вычислить интеграл для $G(z)$. Более того, дифференциальное уравнение также оказывается весьма запутанным, причем его степень растет как степень полинома $V(x)$. Однако существует универсальная форма уравнения для резольвенты, которую мы сейчас обсудим. Чтобы ее найти, рассмотрим следующий по простоте пример кубического потенциала. Тогда, как и выше, можно написать

$$
0=\int_{C} d x \frac{\partial}{\partial x}\left(\frac{e^{x^{3} / 3-T_{1} x}}{z-x}\right)
$$

что эквивалентно

$$
G^{\prime}(z)=V^{\prime}(z) G(z)-\int_{C} d x x e^{x^{3} / 3-T_{1} x}-4 z \int_{C} d x e^{x^{3} / 3-T_{1} x} .
$$

В противоположность гауссовому случаю, моменты $e^{x^{3} / 3-T_{1} x}$ вычислить невозможно. Более того, эти моменты содержат неоднозначность, связанную с выбором контура интегрирования.

\footnotetext{
${ }^{3)}$ Следует проявить аккуратность, интегрируя вокруг точки $z=x$.
} 
Однако заметим, что невычислимая часть является линейным полиномом. Следовательно, мы получаем уравнение

$$
G^{\prime}(z)=\left[V^{\prime}(z) G(z)\right]_{-}
$$

где $[. . .]_{-}$обозначает проектор на отрицательные степени $z$, и мы учли, что $\left[G^{\prime}(z)\right]_{-}=G^{\prime}(z)$. Глядя на уравнение $(22)$, заметим, что оно также удовлетворяет (25). Более того, повторяя вывод для общего полиномиального потенциала, мы приходим к тому же универсальному результату (25).

\section{3. СЛУЧАЙ МАТРИЦ $N \times N$}

Теперь мы рассмотрим настоящий матричный интеграл, в основном следуя той же линии обсуждения, что и в предыдущей главе.

3.1. Петлевые уравнения. Начнем с интеграла (1), снова не фиксируя контур интегрирования. (Так как это многократный интеграл, у нас имеется свобода в выборе своего контура для каждого интегрирования.) Получим вначале набор уравнений, которому удовлетворяет этот интеграл. Как и ранее, мы рассматриваем интегралы от полной производной,

$$
\int D M \operatorname{tr}\left[\frac{\partial}{\partial M^{\mathrm{t}}}\left(M^{n+1} \exp \left(\operatorname{tr} V(M)+\sum_{k} t_{k} \operatorname{tr} M^{k}\right)\right)\right]=0
$$

где $M^{\mathrm{t}}$ - транспонированная матрица. Эти уравнения приводят к следующему набору условий (или уравнений Швингера-Дайсона, или тождеств Уорда, или условий Вирасоро) [12]:

$$
\hat{L}_{m} Z_{N}(t)=0, \quad m \geqslant-1, \quad \hat{L}_{m}=\sum_{k \geqslant 0} k\left(t_{k}\right) \frac{\partial}{\partial t_{k+m}}+\sum_{\substack{a+b=m \\ a, b \geqslant 0}} \frac{\partial^{2}}{\partial t_{a} \partial t_{b}}
$$

где $\partial Z_{N} / \partial t_{0} \equiv N Z_{N}$. Заметим, что на этот раз мы не можем заменить производные по старшим константам связи на высшие производные по первой константе связи. Как и ранее, для получения нетривиальных решений этих уравнений нужно сдвинуть первые $p$ констант связи: $t_{k} \rightarrow T_{k}+t_{k}, k=1, \ldots, p$, и затем работать со статистической суммой (1) как со степенны́м рядом по константам связи $t_{k}$. Тогда к условиям (27) нужно добавить также условия

$$
\frac{\partial Z_{V}}{\partial T_{k}}=\frac{\partial Z_{V}}{\partial t_{k}} \quad \forall k=0, \ldots, n+1 .
$$

Однако теперь у нас имеется набор уравнений с производными по разным константам связи, которые не сводятся к одному обыкновенному дифференциальному уравнению, скажем, по $T_{1}$. Следовательно, свобода в решении уравнений $(27)$ много больше. 
3.2. Матричная модель. Как много решений уравнения (27) мы теперь ожидаем получить? Чтобы понять это, снова изучим сверхупрощенный пример. Посмотрим на модель, подобную (2), но без определителя Вандермонда. Такая статистическая сумма сводится к произведению независимых факторов:

$$
Z_{N}(T \mid t)=\prod_{i} \int_{C_{i}} d x_{i} \exp \left(\sum_{k} t_{k} x_{i}^{k}\right),
$$

каждый из которых является решением соответствующего обыкновенного дифференциального уравнения. Скажем, в кубическом случае

$$
Z_{N}(T \mid t)=\prod_{i}^{N}\left[\xi_{i} \operatorname{Ai}(\eta)+\zeta_{i} \operatorname{Ai}_{2}(\eta)\right]=\sum_{k} \Xi_{k}[\operatorname{Ai}(\eta)]^{k}\left[\operatorname{Ai}_{2}(\eta)\right]^{N-k}
$$

где $\Xi_{k}$ - сконструированные из произведений $\xi_{i}$ и $\zeta_{i}$ коэффициенты, которые определяют произвол в статистической сумме матричной модели. Так как $\Xi_{k}$ - произвольные коэффициенты, можно их интерпретировать как аналоги коэффициентов Фурье в разложении Фурье произвольной функции $Z_{N}(\eta)$ переменной $\eta$, где экспоненты Фурье заменены комбинацией функций Эйри.

В общем случае потенциала степени $p$, как обсуждалось в предыдущей главе, имеется $p-1$ независимых базисных функций, т.е. произвол в статистической сумме матричной модели есть произвольная функция $p-1$ переменной. Это же, конечно, верно для модели (2), в которой имеется определитель Вандермонда.

Поймем теперь источник возникновения произвольной функции $p-1$ переменной в терминах условий (27). С этой целью заметим, что если решать эти условия рекуррентно, раскладывая статистическую сумму в степенно́й ряд по константам связи $t_{k}$ (подобно (7)), только первые два условия (27) действительно ограничивают $Z_{N}(T)$, в то время как все остальные условия просто позволяют рекуррентно восстанавливать зависимость от констант $t_{k}$. Как мы уже объясняли, условия (27) менее ограничительны, чем (5), так как они не сводятся к обыкновенному дифференциальному уравнению по $T_{1}$. Первые два условия (27) линейны по производным, и, следовательно, мы согласованно ограничим их на $t=0$ и затем выразим две производные, например $\partial Z / \partial T_{p+1}$ и $\partial Z / \partial T_{n}$, через $\partial Z / \partial T_{l}$ с $l=0, \ldots, p-1$. Как следствие, статистическая сумма может быть представлена в виде

$$
Z_{N}(T)=\int d k z\left(k ; \eta_{2}, \ldots, \eta_{p}\right) e^{\left(k x-k^{2} w\right) / \hbar}
$$

с произвольной функцией $z$ от $p$ аргументов $\left(k, \eta_{2}, \ldots, \eta_{p}\right)$. Здесь использованы следующие переменные, инвариантные по отношению к действию первого условия:

$$
\begin{gathered}
w=\frac{1}{p+1} \ln T_{p+1}, \quad x=T_{0}+\cdots \sim \eta_{p+1}, \\
\eta_{k}=\left(T_{p}^{k}+\frac{k(k-2) !}{p !} \sum_{l=1}^{k-1}(-)^{l} \frac{(p+1)^{l}(p-l) !}{(k-l-1) !} T_{p-l} T_{p}^{k-l-1} T_{p+1}^{l}\right) T_{p+1}^{-k p /(p+1)} .
\end{gathered}
$$

Мы обсуждали частный случай этих формул в п. 2.3 . 
3.3. Матричный интеграл. Обсудим теперь, каким мог бы быть выбор базисных матричных интегралов. Мы в основном повторяем процедуру, которую применяли в случае однократного интеграла, т.е. используем метод перевала. Различные перевальные точки $M=M_{0}$ даются уравнением $V^{\prime}\left(M_{0}\right)=0$. Если полином

$$
V^{\prime}(x)=\prod_{i=1}^{p}\left(x-\alpha_{i}\right)
$$

имеет корни $\alpha_{i}$, тогда, поскольку $M_{0}$ - матрицы, определенные по модулю $U(N)$ сопряжений (что позволяет диагонализовывать любую матрицу и переставлять ее собственные значения), различные перевальные точки имеют вид

$$
M_{0}=\operatorname{diag}\left(\alpha_{1}, \ldots, \alpha_{1} ; \alpha_{2}, \ldots, \alpha_{2} ; \ldots ; \alpha_{p}, \ldots, \alpha_{p}\right),
$$

причем $\alpha_{i}$ появляется $N_{i}$ раз, $\sum_{i=1}^{p} N_{i}=N$. В действительности нет нужды сохранять эти $N_{i}$ неотрицательными целыми числами - в окончательных формулах они могут быть заменены любым комплексным числом. Более того, $N_{i}$ могут зависеть от $T_{k}$ (т.е. от формы потенциала $V(M)$ ).

Теперь, используя на промежуточной стадии представление матричных интегралов в виде интегралов по собственным значениям, можно переписать [4], [7] матричный интеграл (1) по матрице $M$ размера $N \times N$ как $p$-матричный интеграл по матрицам $M_{i}$ размера $N_{i} \times N_{i}$ (каждая из которых получается сдвигом на $\alpha_{i}$ - это просто замена переменных в матричном интеграле (1)), который является не чем иным, как многоразрезным решением матричной модели [2]:

$$
\begin{aligned}
Z_{V}\left(t \mid M_{0}\right) \sim \int & \prod_{i=1}^{p} D M_{i} \exp \left(\sum_{i, k} \operatorname{tr} t_{k}^{(i)} M^{k}\right) \prod_{i<j}^{p} \alpha_{i j}^{2 N_{i} N_{j}} \times \\
& \times \exp \left(2 \sum_{k, l=0}^{\infty}(-)^{k} \frac{(k+l-1) !}{\alpha_{i j}^{k+l} k ! l !} \operatorname{tr}_{i} M_{i}^{k} \operatorname{tr}_{j} M_{j}^{l}\right)
\end{aligned}
$$

Переменные $t_{k}^{(i)}$ заданы соотношением

$$
\sum_{k=0}^{\infty} t_{k}\left(\sum_{i=1}^{p} \operatorname{tr}_{i}\left(\alpha_{i}+M_{i}\right)^{k}\right)=\sum_{i=1}^{p}\left(\sum_{k=0}^{\infty} t_{k}^{(i)} \operatorname{tr}_{i} M_{i}^{k}\right)
$$

с произвольными матрицами $M_{i}$ размера $N_{i} \times N_{i}$.

Таким образом, в конце концов мы имеем набор базисных функций, которые могут быть описаны подходящим выбором контуров интегрирования и ассоциированы с разными решениями перевального уравнения.

3.4. Резольвента и петлевое уравнение. Другая форма условий (27) получается переписыванием бесконечного набора через единственную производящую функцию всех односледовых корреляторов

$$
\rho^{(1)}(z \mid t) \equiv \widehat{\nabla}(z) \mathcal{F}, \quad \widehat{\nabla}(z) \equiv \sum_{k \geqslant 0} \frac{1}{z^{k+1}} \frac{\partial}{\partial t_{k}}, \quad \mathcal{F} \equiv g^{2} \ln Z_{V}
$$


Вводя обозначение $v(z)$ для $\sum_{k} t_{k} z^{k}$, получаем петлевое уравнение [11]

$$
\begin{aligned}
{\left[V^{\prime}(z) \rho^{(1)}(z \mid t)\right]_{-} } & =V^{\prime}(z) \rho^{(1)}(z \mid t)-\left[V^{\prime}(z) \rho^{(1)}(z \mid t)\right]_{+}= \\
& =\left(\rho^{(1)}(z \mid t)\right)^{2}+\left[v^{\prime}(z) \rho^{(1)}(z \mid t)\right]_{-}+g^{2} \widehat{\nabla}(z) \rho^{(1)}(z \mid t) .
\end{aligned}
$$

Для того чтобы рассмотреть (связные) многоследовые корреляторы, нужно ввести старшие производящие функции (называемые также петлевым средним, резольвентой и т.д.)

$$
\rho^{(m)}\left(z_{1}, \ldots, z_{m} \mid t\right) \equiv \widehat{\nabla}\left(z_{1}\right) \ldots \widehat{\nabla}\left(z_{m}\right) \mathcal{F}
$$

Заметим, что $G(z)$, введенная в выражении $(10)$, равна $Z(T \mid t) \rho^{(1)}(z \mid t)$. Более того, при всех $t_{k}=0$ уравнение (39) сводится к $(25)$. Однако величина $\rho^{(1)}(z \mid 0)$ генерирует только корреляторы односледовых операторов (моменты), чего недостаточно в матричном случае. Следовательно, в этом случае нужно знать полную величину $\rho^{(1)}(z \mid t)$. Она может быть выражена, однако, через $\rho^{(m)}\left(z_{1}, \ldots, z_{m} \mid 0\right)$,

$$
\rho^{(1)}(z \mid t)=\sum_{m \geqslant 0} \frac{1}{m !} \oint \ldots \oint v\left(z_{1}\right) \ldots v\left(z_{m}\right) \rho^{(m+1)}\left(z, z_{1}, \ldots, z_{m} \mid 0\right) .
$$

\section{4. РЕШЕНИЕ РОДА НОЛЬ}

Можно решать петлевые уравнения (39) рекуррентно (см., например, [8]), разлагая резольвенту в сумму $\rho^{(m)}\left(z_{1}, \ldots, z_{m} \mid 0\right)$, а эти последние в ряды по $g$. Это дает двойные рекуррентные соотношения (по $m$ и по порядку по $g$ ). Заметим, что решения этих рекуррентных соотношений в лидирующем порядке можно немедленно получить. Действительно, опуская последний член в уравнении (39), получаем

$$
\rho^{(0 \mid 1)}(z)=\frac{V^{\prime}(z)-y(z)}{2}
$$

(где первый верхний индекс 0 означает лидирующий порядок по $g$ ) с

$$
y^{2}(z)=\left(V^{\prime}(z)\right)^{2}-4 P_{p-1}(z),
$$

где $P_{p-1}(z) \equiv\left[V^{\prime}(z) \rho^{(1)}(z \mid 0)\right]_{+}$- полином степени $p-1$. Коэффициенты этого полинома зависят от $T_{k}$ и первых производных произвольной функции $p-1$ переменной, которая параметризует решения условий (27) (или петлевых уравнений).

Заметим, что формула (43) задает гиперэллиптическую риманову поверхность рода $p-1$, так как отображение (43) задает $p$ разрезов на комплексной плоскости (это и есть то самое многоразрезное решение, которое мы обсуждали во введении). Оказывается, что, по крайней мере, при некоторой специальной фиксации неоднозначности в решениях петлевых уравнений можно сконструировать (мульти)резольвенты как дифференциалы, заданные на этой римановой поверхности [14], [17]. Эта специальная фиксация задается условиями

$$
\oint_{A_{i}} \rho^{(m+1)}\left(z, z_{1}, \ldots, z_{m} \mid 0\right) d z=0
$$


для всех (мульти)резольвент за исключением $\rho^{(0 \mid 1)}(z)$,

$$
\oint_{A_{i}} \rho^{(0 \mid 1)}(z)=S_{i},
$$

где $S_{i}$ - произвольные константы, не зависящие от $T_{k}$, а $A_{i}-A$-циклы на римановой поверхности (43). Константы $S_{i}$ могут быть на самом деле ассоциированы (с точностью до фактора $g$ ) с $N_{i}$ из (36). Более того, базисные функции (36) в точности приводят к условиям (44), (45), ассоциированым также с системой ЗайбергаВиттена-Уизема, соответствующей римановой поверхности (43) [13].

Здесь мы не будем углубляться в дальнейшие детали, отсылая читателя к работам [10] и [14] за полученными недавно результатами и ссылками. Заметим только, что условия (44), (45) выделяют специальные решения, которые выживают (гладко себя ведут) при изменении числа $p$ констант связи $T_{k}$ аналогично тому, что мы наблюдали в п. 2.3. Действительно, для любого числа $q$ ненулевых $T_{k}$ можно потребовать, чтобы комплексная кривая (риманова поверхность) имела вид

$$
y^{2}(z)=\left(V^{\prime}(z)\right)^{2}-4 P_{q-1}(z)=H_{q-p}^{2}(z) R_{2 p}(z),
$$

где $H_{q-p}^{2}(z)$ и $R_{2 p}(z)$ - полиномы. Следовательно, независимо от $q$ имеется гиперэллиптическая кривая $\tilde{y}^{2}(z)=R_{2 p}(z)$ рода $p-1$. Более того, произвол, который имеется в матричной модели в этом случае, задается $p-1$ константой $S_{i}$ (детали см. в работе [14]). Ключевое различие со случаем, рассмотренным в п. 2.3, заключается в том, что, когда имеешь дело с простым интегралом, можно вычислять старшие моменты, дифференцируя этот интеграл по низшим константам связи. В то же время в матричном случае так делать нельзя из-за различия следов степеней матриц и степеней следов. Следовательно, технически очень удобно добавлять следы старших степеней матриц прямо в матричный потенциал. Однако при этом надо быть осторожным и не изменить такой процедурой выбранного решения матричной модели. Это в точности достигается сохранением кривой $\tilde{y}(z)(46)$.

Благодарности. Автор благодарен А. Александрову, Д. Васильеву, А. Маршакову, А. Морозову и Л. Чехову за многочисленные обсуждения и В. Долотину за комментарии к тексту. Работа частично поддержана федеральной программой российского Министерства промышленности, науки и технологии (грант № 40.052.1.1.1112) РФФИ (грант №04-01-00646а) и Программой поддержки ведущих научных школ (грант № 96-15-9679).

\section{Список литературы}

[1] F. Cachazo, K. Intriligator, C. Vafa. Nucl. Phys. B. 2001. V. 603. P. 3; hep-th/0103067; F. Cachazo, C. Vafa. $N=1$ and $N=2$ geometry from fluxes. hep-th/0206017.

[2] R. Dijkgraaf, C. Vafa. Nucl. Phys. B. 2002. V. 644. P. 3; hep-th/0206255; V. 644. P. 21; hep-th/0207106; A perturbative window into non-perturbative physics. hep-th/0208048. 
[3] K. Demeterfi, N. Deo, S. Jain, C.-I Tan. Phys. Rev. D. 1990. V. 42. P. 4105; J. Jurkiewicz. Phys. Lett. 1990. V. 245. P. 178; C. Crnković, G. Moore. Phys. Lett. B. 1991. V. 257. P. 322; G. Akemann, J. Ambjørn. J. Phys. A. 1996. V. 29. P. L555; cond-mat/9606129.

[4] G. Bonnet, F. David, B. Eynard. J. Phys. A. 2000. V. 33. P. 6739.

[5] S. Kharchev, A. Marshakov, A. Mironov, A. Morozov. Nucl. Phys. B. 1993. V. 397. P. 339; hep-th/9203043.

[6] M. L. Mehta. Random Matrices. New York: Acad. Press, 1990.

[7] A. Klemm, M. Marino, S. Theisen. JHEP. 2003. V. 0303. P. 051; hep-th/0211216.

[8] A. Alexandrov, A. Mironov, A. Morozov. Int. J. Mod. Phys. A. 2004. V. 19. P. 4127; hep-th/0310113.

[9] A. Alexandrov, A. Mironov, A. Morozov. Unified description of correlators in nonGaussian phases of Hermitean matrix models. hep-th/0412099.

[10] A. Alexandrov, A. Mironov, A. Morozov. Fortschr. Phys. 2005. V. 53. P. 512; hep-th/0412205.

[11] A. A. Migdal. Phys. Rep. 1983. V. 102. P. 199; J. Ambjørn, J. Jurkiewicz, Yu. Makeenko. Phys. Lett. B. 1990. V. 251. P. 517; Yu. Makeenko. Mod. Phys. Lett. A. 1991. V. 6. P. 1901.

[12] A. Gerasimov, A. Marshakov, A. Mironov, A. Morozov, A. Orlov. Nucl. Phys. B. 1991. V. 357. P. 565; F. David. Mod. Phys. Lett. A. 1990. V. 5. P. 1019; A. Mironov, A. Morozov. Phys. Lett. B. 1990. V. 252. P. 47; J. Ambjørn, Yu. Makeenko. Mod. Phys. Lett. A. 1990. V. 5. P. 1753; H. Itoyama, Y. Matsuo. Phys. Lett. B. 1991. V. 255. P. 202.

[13] L. Chekhov, A. Mironov. Phys. Lett. B. 2003. V. 552. P. 293; hep-th/0209085; L. Chekhov, A. Marshakov, A. Mironov, D. Vasiliev. Phys. Lett. B. 2003. V. 562. P. 323; hep-th/0301071.

[14] L. Chekhov, A. Marshakov, A. Mironov, D. Vasiliev. Complex geometry of matrix models. hep-th/0506075.

[15] Справочник по специальным функциям с формулами, графиками и математическиим таблицами. Ред. М. А. Абрамовиц, И. А. Стиган. М.: Наука, 1979.

[16] Г. Бейтмен, А. Эрдейи. Высшие трансцендентные функции. Т. 2. М.: Наука, 1974.

[17] B. Eynard. JHEP. 2004. V. 0411. P. 031; hep-th/0407261. 